Case Report

\title{
CAVERNOUS HAEMANGIOM A IN THE GASTROCNEMIUS MUSCLE: A RARE PRESENTATION IN THE GERIATRIC AGE GROUP
}

\author{
H. Ravindranath Rai ${ }^{1}$, Arjun Ballal ${ }^{2}$, Keerti Mohan ${ }^{3} \&$ Rajsankar N.R. ${ }^{4}$ \\ ${ }^{1}$ Professor \& HOD, ${ }^{2,3,4}$ P.G. Students, Department of Orthopaedic Surgery, K.S. Hegde Medical Academy, \\ Nitte University, Mangalore - 575 018, Karnataka, India. \\ Correspondence: \\ Arjun Ballal \\ P.G. Student, Department of Orthopaedic Surgery, K.S. Hegde M edical Academy, \\ Nitte University, M angalore - 575018, Karnataka, India. \\ Mobile : +91 8431644077 E-mail : Arjchess_Lp@ rediffmail.com
}

\begin{abstract}
:
Intramuscular haemangiomas are believed to be hamartomatous and are a distinctive type of haemangioma occurring within skeletal muscle. They account for less than $1 \%$ of all haemangiomas. They occur more often in trunk and extremity muscles.

A sixty five year old lady presented with swelling and pain from the back of the left kneefor two years.

On clinical examination, a mass of about 10X10 cms in size was noted in the left popliteal fossa. The swelling was tender with well defined borders but fixed to the muscle. She had a flexion deformity of knee of ten degrees.

M RI revealed a large encapsulated cystic lesion in the posterior aspect of the muscular compartment of the knee.

She underwent excision of the mass, intraoperatively the mass was noted to be arising from the gastrocnemius. Histopathology revealed large cavernous filled spaces filled with blood which indicated a cavernous haemangioma.

M ost of the literature suggest the occurance of capillary haemangioma to be a commoner one. Occurance of a cavernous haemangioma is usually before the third decade and is congenital in most times. M ost of the authors emphasize that radiological methods are generally insufficient for the correct diagnosis of intramuscular hemangiomas, and surgery is the treatment of choice to exclude malignancy and for adequate treatment of these lesions.

Hence, the present case which we are reporting here is a rare occurance of a cavernous haemangioma of the gastrocnemius in a 65 year old lady, which was managed by surgical methods.
\end{abstract}

Keywords : cavernous haemangioma, gastrocnemius, popliteal fossa, intramuscular haemangiomas, blood filled spaces

\section{Introduction:}

Haemangiomas are benign vascular neoplasms characterized by an abnormal proliferation of blood vessels that most often occur in the skin or subcutaneous tissue. Haemangiomas are the most common benign soft tissue tumour of infancy and childhood comprising $7 \%$ of all soft tissue tumours. ${ }^{3}$ Intramuscular haemangiomas account for $0.8 \%$ of all haemangiomas. ${ }^{3.4}$

\begin{tabular}{|c|}
\hline Access this article online \\
\hline Quick Response Code \\
\hline
\end{tabular}

Capillary haemangiomas are more common in muscle than cavernous and compound types. ${ }^{2}$

The etiology is unclear but possibly congenital in origin. $^{3-7}$ Haemangiomas commonly occur in the skin or subcutaneous tissues followed by the deep tissues, occasionally are intramuscular and rarely within bone.

It has been estimated that $90 \%$ of intra-muscular haemangiomas occur before the third decade of life. ${ }^{1}$

There is a general agreement that females are more commonly affected than males. ${ }^{4,8}$

Hemangiomas in muscle tissue can develop at any age, but most often occur in young adults. Capillary hemangiomas are more common in muscle than cavernous and compound types. Any muscle can be involved. Because they are located within the muscle, these hemangiomas often show no visible signs, although some may cause swelling in the area of the tumor that increases with activity. ${ }^{2}$ 


\section{Case Report:}

A sixty five year old lady presented to us with complaints of pain and swelling over the posterior aspect of the left calf muscle since two years. She also complained to have difficulty in climbing stairs and walking uphill due to the pain and swelling.

The pain was insidious in onset and progressive in nature and aching type. There was no history of any trauma or fall. No history suggestive of any sickness in the past or present. No history of any medical complaints. She also had no history suggestive of any neurovascular deficits over the limb.

On examination: The swelling measured about $10 \times 10 \mathrm{cms}$ over the popliteal fossa and the proximal calf muscles of the left leg. The skin over the swelling was shiny looked normal. No visible scars or sinuses were present (Figure: 1).

The swelling was firm in consistency, non-mobile. The swelling was not attached to the skin, but attached to the muscle. The range of motion was 10-60 degrees flexion. No abnormalities were detected in the tests for the patella, the menisci and the cruciate ligaments.

Radiologically: the X-rays revealed a mass over the posterior aspect of the knee with minimal calcifications and osteophytes [Figures 2(A), (B)].

The M RI reported as a large cystic lesion in the posterior aspect of the muscular compartment of the knee, internal solid and fluid areas which is ioshyperintense. [Figure: 3(A), (B)]

The patient underwent an FNAC of the mass whose reports were inconclusive. This was followed by a biopsy which revealed large areas of coagulative necrosis, fibrocartillagenous adipose tissue and haemorrhage. Repeat biopsy was suggested.

The patient then underwent an excision biopsy of the mass at a later date. On surgical exposure (Figure 4) a brownish mass arising from the medial head of gastrocnemius measuring around $20 \times 20 \mathrm{cms}$ (Figure 5) separate from all attachments with fluid. The biopsy reported large cavernous spaces filled with blood, lined by flattened endothelial cells and intercommunicating channels. [Figures6 (A), (B)]

Post operatively she was started on antibiotics and knee exercises. Slowly her range of motion of knee improved and reached almost full range at six weeks review with normal radiography.

\section{Discussion:}

The term "haemangioma" is commonly misused to describe any type of vascular abnormality, including vascular malformation, in both medical and surgical fields. ${ }^{9}$ Hassanein et al. revealed in a study that the term was incorrectly used in 71.3 percent of publications on the Pubmed database. ${ }^{10}$

Venous malformation can occur in every muscle group with pain and swelling being the usual complaints. Vascular malformations are usually present at birth, grows proportionally with the child and never involutes. They can be classified as arterial, arteriovenous, venous, capillary or lymphatic. Haemangiomas are distinguished by endothelial hyperplasia, multilaminated basement membrane formation beneath the endothelium and clinical history of rapid growth during infancy. ${ }^{11}$ Additionally, intramuscular venous malformation can be distinguished from cavernous haemangiomas the former has no regression phase and certain M RI characteristics.

Histologically, haemangiomas are classified based on the pre- dominant type of vascular channel asfollows ${ }^{12}$ :

1. Capillary haemangioma, which are composed of small vessels lined by flattened endothelium. It is the commonest type and subdivided into juvenile, verrucous and senile type.

2. Cavernous haemangioma, which are composed of dilated, blood filled spaces, lined by flattened endothelium. There is abundant adipose tissue and they do not involute as the capillary haemangioma.

3. Arteriovenous haemangioma, which is characterized by the presence of fetal capillary bed with abnormal communication of the arteries and veins.

4. Venous haemangioma, which is composed of thick walled vessels containing muscle. 


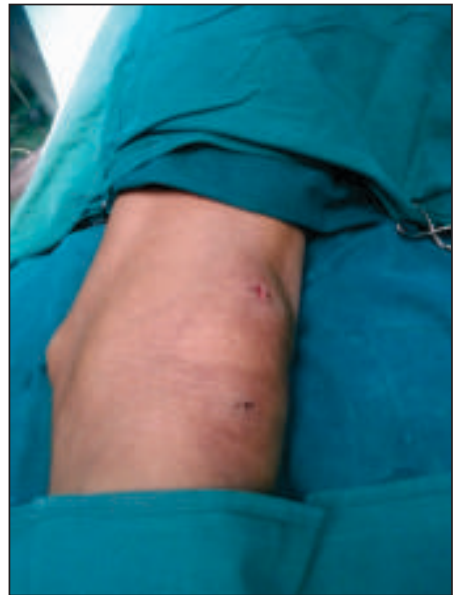

FIGURE 1: Approximately $10 \times 10 \mathrm{~cm}$ swelling over the popliteal fossa of the left leg. The puncture wound isfrom the biopsy.
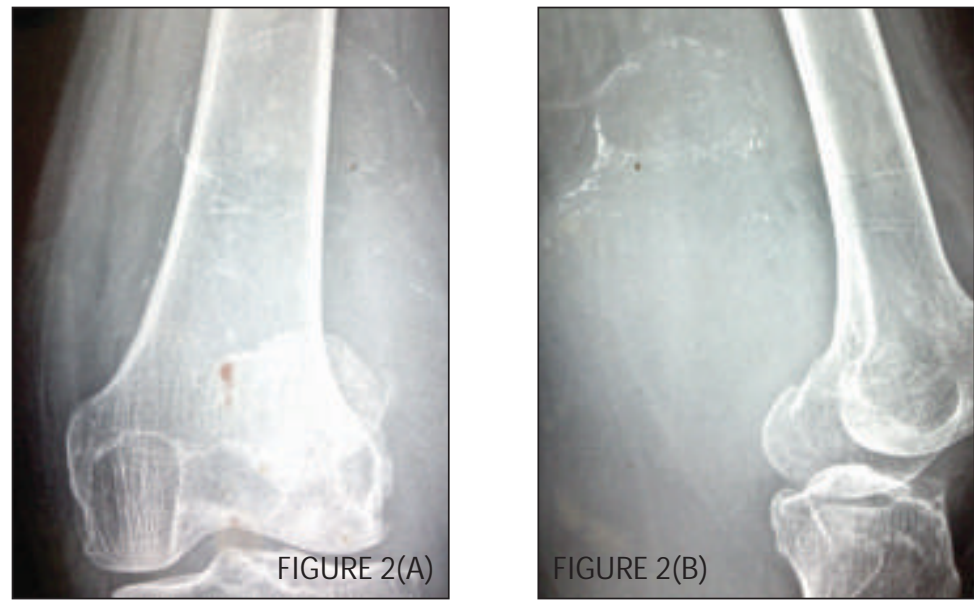

FIGURE 2(A),(B) : X-ray left knee AP and lateral views showing thin posterior calcifications.

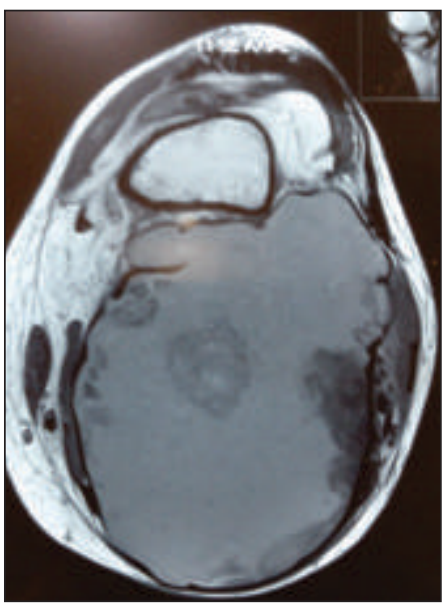

FIGURE 3(A): Axial section of MRI left knee showing the fluid filled cystic lesion.

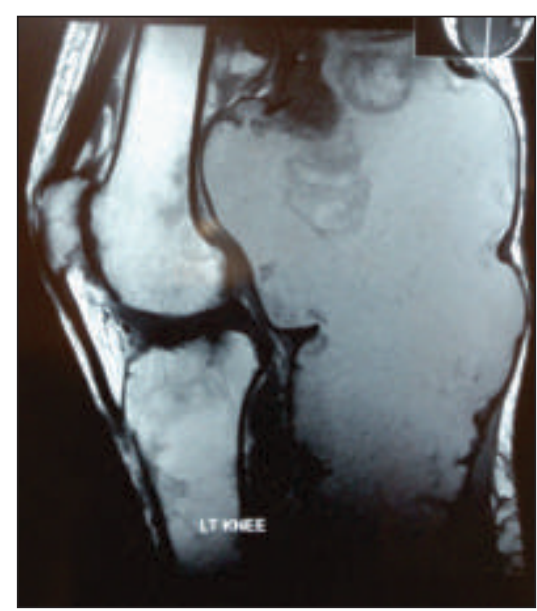

FIGURE 3(B): Coronal section of MRI showing the cysticlesion in the posterior compartment of knee.

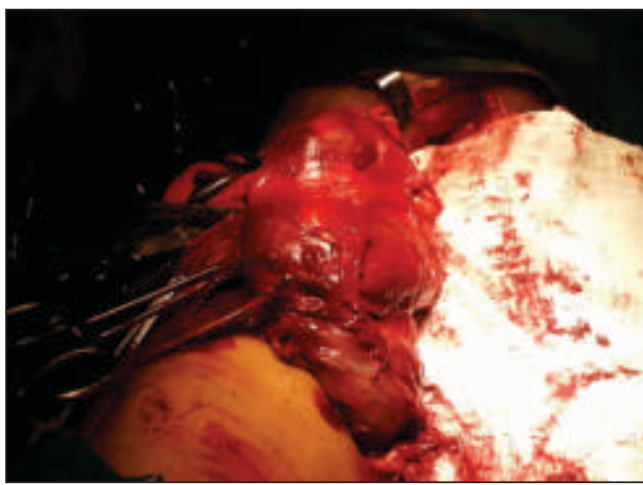

FIGURE 4: Surgical exposure of the mass



FIGURE 5: Mass measuring 20X20 cms over the medial head of gastrocnemius
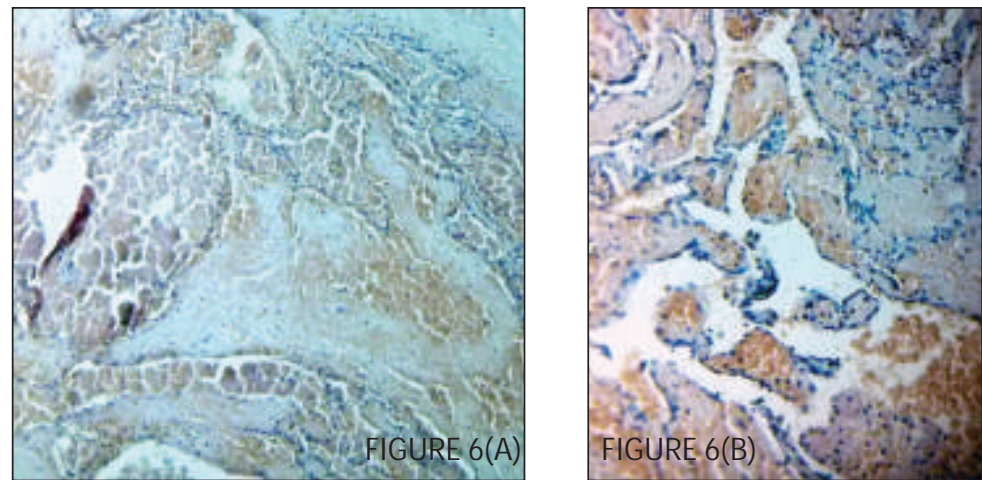

FIGURE 6 (A) : Histopathology showing large cavernous spaces filled with blood (H\&E, X100)

FIGURE 6 (B) : Histopathology showing cavernous spaces lined by flattened endothelial cells and intercommunicating channels(H\&E,X400) 
The latter occur most commonly in young adults with80-90\% presenting in individuals younger than 30 years. ${ }^{3,4}$ Pain is a cardinal symptom in $60 \%$ of the cases, ${ }^{3}$ with the lower extremity being the commonest site of involvement. The quadriceps is the most frequently affected muscle. ${ }^{13,14}$

Haemangioma confined to the calf muscle can cause wasting and contracture. Intramuscular haemangiomas progressively enlarge but never metastasize. ${ }^{5}$ There is a $9 \%$ recurrence rate after surgical excision. ${ }^{3}$ Periosteal reaction adjacent to the haemangioma may mimic osteomyelitis or bone tumour. The plain radiograph may show flecks of calcification which are uncommon but highly suggestive of haemangioma.

$M R I$ is important for further characterization of the substance and extentof the soft tissue haemangioma. On T1-weighted images, a haemangioma appears a slow-tointermediate signal intensity mass with peripheral high signal intensity due to fat overgrowth. On T2-weighted

\section{References:}

1. T Bucci, F De Giulio, A Romano, L Insabato, and L Califano. Cavernous haemangioma of the temporalis muscle: case report and review of the literature. Acta Otorhinolaryngol Ital. 2008 April; 28(2): 83-86.

2. Orthoinfo.aaos.org/topic. cfm?topic $=A 00630$ ?

3. AllanPW, EnzingerFM .Haemangiomaofskeletalmuscle:ananalysisof 89 cases. Cancer 1972;29:8-22.

4. WatsonWL, McCarthyWD. Bloodandlymphvesseltumors. Areportof 1056 cases. Surg Gynecol Obstet 1940;71:569-88.

5. Ferguson ILC. Haemangiomata of skeletal muscle. Br J Surg 1972;598:634-7.

6. JonesKG. Cavernoushaemangioma ofstriatedmuscle areviewofliterature and are port off our cases. J Bone Joint Surg 1953;35A:717-28.

7. Mencke HJ, Zilkens], BigalkeKH, AmmonJ. The problem of intramuscular haemangioma. Arch Orthop Trauma Surg 1982;100:243-7.

8. BehamAJ,Fletcher CDM. Intramuscular angioma:a clinicopathologic analysis of 74cases. Histopathology 1991;18:53-9.

9. Mulliken J B,G Lowackil. Haemangiomas and vascular malformations in infants and children: a classification based on endothelial characteristics. Plast Reconstr Surg 1982;69:412-22. images, it shows areas of high signal intensity due to vascular tissue and intermediate signal intensity due to fat.MRI shows venous malformations to be isotense to surrounding muscle on T1-weighted and hyperintense on T2-weighted images. ${ }^{9}$

T Bucci et al reported a case of cavernous haemangioma of the temporalis muscle in $2008 .{ }^{1}$

Hristov N, Atanasov Z, Zafirovski G, Mitrev Z reported a case of Intramuscular cavernous hemangioma in the left soleus muscle which underwent a successful surgical resection. ${ }^{15}$

Dong Hwee Kim et al, reported a case of an intramuscular cavernous haemangioma which mimicked a case of myofascial pain syndrome. ${ }^{16}$

Hence, a clear knowledge and understanding of these conditions are very much essential to understand and manage such conditions.

10. Hassanein AH, Mulliken JB, Fishman SJ, Greene AK. Evaluation of terminology for vascular anomalies in current literature. Plast Reconstr Surg 2011;127(1):347-51.

11. Fernandez-Pineda I. Intramuscular cavernous haemangioma. Am J Surg 2009;198(2):300-1.

12. HeinKD, Mulliken JB, Kozakewich HP, Upton J, Burrows PE. Venous malformations of skeletal muscle. Plast Reconstr Surg 2002;110:1625-35.11.

13. BuetowPC, Kransdorf MJ,M oserJrRP,JelinekJS,BerreyBH.Radiographic appearance ofintramuscularhemangiomawithemphasison M Rimaging. Am J Roentgenol 1990;154:563-7.

14. ChristensonJT, GunterbergB. Intramuscularhaemangiomao ftheextremities: is computerizedtomographyuseful? $\mathrm{Br}$ JSurg $1985 ; 72: 748-50$.

15. Hristov N, Atanasov Z, Zafirovski G, M itrev Z. Intramuscular cavernous hemangioma in the left soleus muscle: successful surgical treatment. Interact Cardiovasc Thorac Surg. 2011 Nov; 13(5):521-2.

16. Dong Hwee Kim, M iriam Hwang, Yoon Kyoo Kang, In Jong Kim and Yoon Kun Park. J Korean M ed Sci. 2007 June; 22(3): 580-582. 